\title{
In vitro analysis of integrin expression during chondrogenic differentiation of mesenchymal stem cells and chondrocytes upon dedifferentiation in cell culture
}

\author{
ULRICH REINHART GOESSLER ${ }^{1}$, KAREN BIEBACK ${ }^{2}$, PETER BUGERT ${ }^{2}$, \\ TOBIAS HELLER $^{1}$, HANEEN SADICK $^{1}$, KARL HÖRMANN $^{1}$ and FRANK RIEDEL ${ }^{1}$ \\ ${ }^{1}$ Department of Otolaryngology, Head and Neck Surgery; ${ }^{2}$ Institute of Transfusion Medicine \\ and Immunology, Red Cross Blood Service of Baden-Württemberg/Hessia, \\ Ruprecht Karls-University Heidelberg, Faculty of Clinical Medicine Mannheim, Germany
}

Received September 9, 2005; Accepted October 28, 2005

\begin{abstract}
Tissue engineering represents a promising method for generating chondrogenic grafts for reconstructive surgery. In cultured chondrocytes, the dedifferentiation of cells seems unavoidable for multiplication. Stem cells, however, displaying unlimited self-renewal and the capacity to differentiate towards chondrocytes, might be usable after further characterization. As the interactions between the extracellular matrix and the cellular compartment can alter the cellular behaviour, we investigated the expression of integrins using microarray analysis during chondrogenic differentiation of human mesenchymal stem cells (MSC) in comparison with dedifferentiatiating human chondrocytes (HC) harvested during septoplasty. During chondrogenic differentiation of MSC, the fibronectin-receptor (Integrin $\beta 1 \alpha 5$ ), fibronectin and the GPIIb/IIIa-receptor were downregulated. The components of the vitronectin-receptor (Integrin $\alpha v 33$ ) and CD47 were constantly expressed and ILK was downregulated. Vitronectin and osteopontin were not expressed by the cells. In HC, Integrin $B 1 \alpha 5$ in conjunction with the ligand fibronectin were upregulated during dedifferentiation, Integrin $\alpha \mathrm{v} \beta 3$ as well as the GBIIb/IIIa-receptor were activated on day 21 but neither vitronectin nor osteopontin were expressed by the cells. The integrins, $\beta 2, \beta 4, \beta 6, \beta 8$ and $\alpha 2, \alpha 4, \alpha 6, \alpha 7, \alpha 11$, were not expressed at any time. ILK, CD47, and ICAP were activated with ongoing dedifferentiation. In conclusion, a candidate for signal-transmission is the fibronectin receptor (integrin $\alpha 5 \beta 1$ ) in conjunction with its ligand fibronectin. Other receptors, e.g. for vitronectin and osteopontin $(\alpha \mathrm{v} 33)$, or their ligands
\end{abstract}

Correspondence to: Dr Ulrich Goessler, Universitäts-HNOKlinik Mannheim, Theodor-Kutzer-Ufer, D-68135 Mannheim, Germany

E-mail: ullrich.goessler@hno.ma.uni-heidelberg.de

Key words: Integrin, mesenchymal stem cells, cartilage, chondrocytes, tissue engineering, dedifferentiation, fibronectin, extracellular matrix do not seem to be involved in signal transmission for dedifferentiation. The GPIIb/IIIa-receptor might assist the process of dedifferentiation. Intracellularly, ILK, ICAP1 and CD47 might be involved in the transduction of integrindependent signals.

\section{Introduction}

In the last few years, the need for chondrogenic grafts in reconstructive surgery has been accretive. Traditional surgical methods for the reconstruction of tissue defects rely on the transplantation of auto- and allogenous tissues or, accordingly, alloplastic replacement with synthetic medical products. The disadvantages of these techniques are the limited availability and donor site morbidity of auto- or allogenic transplants (1). The overwhelming shortage of donor cells and tissue is a major problem in reconstructive medicine. This problem could be alleviated through the use of autologous adult stem cells for allogenic transplantation (2,3). Stem cells are distinguished from progenitor cells by their capacity for both self-renewal and multilineage differentiation (4). It is this capacity for self-renewal that makes stem cells particularly useful for reconstructive surgery because, in theory, they could provide an unlimited source of autologous donor material for transplantation $(5,6)$.

The fundamental premise of tissue engineering is the regeneration of tissue and restoration of organ function through implanting cells/tissue grown ex vivo or stimulating cells to grow into an implanted matrix (7). Tissue engineering involves the use of cells coupled with biological or artificial matrices which guide the cells during tissue repair or regeneration (8). These cells can be driven by specific bioactive molecules, ex vivo gene transfer and other physical factors to form neotissues in vitro for future reimplantation in vivo. Alternatively, cells and special matrices, which can include bioactive molecules such as growth factors, can be combined in vivo to attempt to enhance tissue repair (9).

The promise of tissue engineering is perhaps most relevant to chondrogenic defects because cartilage has little self-healing potential. Numerous in vitro and in vivo investigations into cartilage engineering have been performed. Cartilage is a 
unique avascular, aneural and alymphatic load-bearing live tissue. One problem in the generation of chondrogenic grafts lies in the dedifferentiation of the cells: loss of the chondrogenic phenotype and development of a more fibroblastoid cellshape. These morphological changes are followed by distinct changes in the patterns of gene expression (10). The integrin family of cell surface receptors appears to play a major role in the mediation of the cell-ECM interactions associated with structural and functional changes in surrounding tissue $(11,12)$. The integrins are heterodimeric glycoproteins that are composed of an $\alpha$ and a $\beta$ subunit, each of which has extracellular and cytoplasmic domains. The extracellular domains bind to a number of ECM proteins, including collagen types II and VI, fibronectin and matrix Gla-protein. Several recent studies have provided evidence that chondrocytes express integrins (13-18). Salter et al used immunohistochemical staining in normal adult articular cartilage, and noted that integrin $\alpha 5 \beta 1$ was the most prominently expressed chondrocyte integrin (17). A more recent study demonstrated that the chondrocyte expression of $\alpha 1 \beta 1, \alpha 5 \beta 1$ and $\alpha v \beta 5$ was accompanied by weak expression of integrins $\alpha 3 \beta 1$ and $\alpha v \beta 3$ (16). The Integrin $\alpha 2 b$ (ITGA2B) gene encodes platelet glycoprotein $\mathrm{IIb}$, the alpha subunit of the platelet membrane adhesive protein receptor complex GPIIb/IIIa. The beta subunit, GPIIIa, is encoded by the ITGB3 gene. The GPIIb/IIIa complex belongs to the integrin class of cell adhesion molecule receptors that share a common heterodimeric structure with $\alpha$ and $\beta$ subunits. The GPIIb/GPIIIa complex acts as a receptor for fibrinogen, von Willebrand factor and fibronectin (19).

Integrin-mediated signaling has so far been characterized to be involved in a variety of cellular processes, such as differentiation, adhesion and migration. Hannigan et al found that Integrin-linked kinase (ILK) coimmunoprecipitated with B-1 integrin from cell lysates and that overexpression of ILK disrupted cell architecture and inhibited adhesion to integrin substrates, suggesting that ILK regulates integrin-mediated signal transduction (20). In addition to ILK, integrin cytoplasmic domain-associated protein 1 (ICAP1) interacts with the cytoplasmic domain of beta-1 integrin (21). CD47 or integrin-associated protein (IAP) is a membrane protein that is involved in the increase in intracellular calcium concentration that occurs upon cell adhesion to the extracellular matrix (22).

As the chondrocyte is responsible for modulating its environment and the chondrocyte phenotype is influenced by the diverse components of the extracellular matrix, the investigation of the molecular basis of distinct changes during developmental processes, especially the process of dedifferentiation for the generation of transplants, might broaden the understanding of impediments in the field of tissue engineering. Investigations of gene-expression patterns of human chondrocytes or mesenchymal stem cells (MSC) during chondrogenic differentiation so far have not been performed. The aim of our study was the analysis of gene-expression patterns of MSC compared to dedifferentiating primary chondrocytes harvested during septoplasty in cell-culture.

\section{Materials and methods}

Cell culture. Human septal cartilage from 32 patients was harvested during septoplasty. The patients gave informed consent and the Faculty of Medicine Mannheim, University of Heidelberg, Germany, approved the study. Cells were isolated using collagenase and pronase. Cell cultures were carried out in Falcon petri dishes at $37^{\circ} \mathrm{C}$ in a $5 \% \mathrm{CO}_{2}$ fully humidified atmosphere using Dulbecco's modified minimum essential medium (DMEM) (Fisher Scientific Co., Pittsburgh, PA, USA) supplemented with $10 \%$ fetal calf serum (FCS) and antibiotics [Life Technologies, Inc. (Gibco BRL), Gaithersburg, MD, USA]. The cells were cultured for 6 and 21 days without growth factors.

Collection and isolation of MSC from bone marrow (BM). Bone marrow was obtained from the femoral shaft of patients undergoing total hip replacement at the orthopedic department of the University Hospital Mannheim. Cells were aspirated into a 5-ml syringe containing CPD anticoagulant. In total, six specimens from female patients were obtained, with the donor age ranging from 68 to 84 years.

To isolate mononuclear cells (MNCs), the bone marrow aspirates were diluted 1:5 with PBS/2 mM EDTA (Nexell, Baxter, Unterschleissheim, Germany; and Merck, Darmstadt, Germany) and carefully loaded onto Ficoll-Hypaque solution (Amersham, Freiburg, Germany). After density gradient centrifugation at $435 \mathrm{x} \mathrm{g}$ for $30 \mathrm{~min}$ at room temperature, MNCs were removed from the interphase and washed two to three times with PBS/EDTA. Cell counts were performed using an automated cell analyzer (Cell-Dyn 3200, Abbott, Wiesbaden, Germany).

BM derived MNCs were set in culture at a density of $1 \times 10^{5} / \mathrm{cm}^{2}$ in $75-\mathrm{cm}^{2}$ tissue culture flasks (Nunc, Wiesbaden, Germany, www.nunc.de) in MSCGM medium (MSCGM BulletKit $^{\mathrm{TM}}$, Cambrex, St. Katharinen, Germany).

After overnight incubation at $37^{\circ} \mathrm{C}$ in a humidified atmosphere containing $5 \% \mathrm{CO}_{2}$, non-adherent cells were removed and fresh medium was added to the flasks. Cultures were maintained and remaining non-adherent cells were removed by complete exchange of culture medium every three to four days. The flasks were screened continuously to obtain developing colonies of adherent cells. Fibroblastoid cells were recovered between days 7 and 10 after initial plating by using $0.04 \%$ Trypsin/0.03\% EDTA (PromoCell, Heidelberg, Germany). Recovered cells were replated at a density of $4000-5000$ cells $/ \mathrm{cm}^{2}$ as passage 1 (P1) cells and thereafter.

Chondrogenic differentiation. To promote chondrogenic differentiation, $2.5 \times 10^{5}$ cells were gently centrifuged $(150 \mathrm{x} \mathrm{g}$, $5 \mathrm{~min}$ ) in a $15-\mathrm{ml}$ polypropylene tube (Greiner) to form a pellet according to the protocol of Mackay et al (23). Without disturbing the pellet, the cells were cultured for four weeks in complete chondrogenic differentiation medium (Cambrex) including $10 \mathrm{ng} / \mathrm{ml}$ TGFB3 (Strathmann Biotec AG, Hamburg, Germany) by feeding twice a week. After the culture period, cryosections were analyzed by Safranin O staining. The sections were fixed with ice-cold acetone (Sigma) and stained with $0.1 \%$ aqueous Safranin O solution (Sigma). Cell nuclei were counterstained with Weigert's iron hematoxylin (Sigma).

For the RNA analysis we harvested and lysed the aggregates in RLT buffer (Qiagen). The lysis was aggravated by freezing the pellet repeatedly in liquid nitrogen. 
Table I. Signal intensities of hybridization signals as measured by the microarray laser scanner and calculated by ArrayVision software during chondrogenic differentiation of MSC.

\begin{tabular}{lccc}
\hline & Signal intensity $($ mean \pm SD) & $\begin{array}{c}\text { Factor of } \\
\text { regulation }\end{array}$ \\
& Undiff & Diff & Undiff vs. Diff \\
\hline B1 & $32134 \pm 3040$ & $15557 \pm 3968$ & 0.58 \\
$\beta 3$ & $2124 \pm 876$ & $869 \pm 359$ & $\mathbf{0 . 4 9}$ \\
$\beta 5$ & $7684 \pm 4811$ & $4344 \pm 2937$ & 0.68 \\
$\alpha 2 b$ & $23892 \pm 9674$ & $30795 \pm 3988$ & 1.55 \\
$\alpha 3$ & $2197 \pm 1016$ & $1154 \pm 464$ & 0.63 \\
$\alpha 5$ & $11790 \pm 4269$ & $9144 \pm 4093$ & 0.93 \\
$\alpha 10$ & $1914 \pm 1450$ & $1191 \pm 513$ & 0.75 \\
$\alpha$ V & $4259 \pm 1788$ & $5257 \pm 1911$ & 1.49 \\
ILK & $21171 \pm 6070$ & $11173 \pm 887$ & 0.63 \\
ICAP-1a & $1616 \pm 315$ & $661 \pm 104$ & $\mathbf{0 . 4 9}$ \\
CD47 & $6147 \pm 3134$ & $5222 \pm 2497$ & 1.02 \\
Fibronectin & $75289 \pm 3543$ & $61995 \pm 2701$ & 0.99 \\
\hline Bold indicas & & &
\end{tabular}

Bold indicates significant up- $(\geq 2.00)$ or downregulation $(\leq 0.50)$.

RNA extraction and microarray hybridization. Extraction of RNA was performed using RNA Mini Kit (Qiagen, Hilden, Germany) according to the manufacturer's protocol and as published previously (24). The RNA concentration was estimated from the absorbance at $260 \mathrm{~nm}$.

Approximately $1 \mu \mathrm{g}$ total RNA was used in each microarray experiment and, for amplification and labeling of mRNA, the SMART technique (SMART fluorescent probe amplification kit; BD Clontech, Heidelberg, Germany) was applied according to the manufacturer's protocol. RNA samples from day 1 were labeled with $\mathrm{Cy} 3$ and day 6 or day 21 samples were labeled with $\mathrm{Cy} 5\left(\mathrm{Cy}^{\mathrm{TM}} 3\right.$ - and $\mathrm{Cy}^{\mathrm{TM}}$ 5-monoreactive dye; Amersham Pharmacia Biotech, Freiburg, Germany). Corresponding Cy3- and Cy5-labeled samples were mixed, vacuum-dried and resuspended in $25 \mu 1$ microarray hybridization buffer (MWG-Biotech; Ebersberg, Germany). Prior to hybridization, the samples were heat-denatured at $95^{\circ} \mathrm{C}$ for 5 min. The human 10K (MWG-Biotech) oligo microarray systems on glass slides were used for mRNA profiling. Hybridization of $\mathrm{Cy} 3 / \mathrm{Cy} 5$-cDNA was performed using cover slips and a hybridization chamber for $16 \mathrm{~h}$ at $42^{\circ} \mathrm{C}$ in a water bath. After stringent washing of the glass slides according to the manufacturer's specifications, the hybridization signals of the $\mathrm{Cy} 3$ and $\mathrm{Cy} 5$ dyes were measured using a microarray laser scanner (GMS418; Affymetrix, MWG-Biotech).

Microarray data analysis and statistics. ArrayVision (Imaging Research, Inc., St. Catharines, ON, Canada) software was used for evaluation and calculation of signal intensities from the raw data images in 16-bit tagged-image-file (TIF) format as described previously. In brief, for evaluation of the hybridization results, we defined a negative $(<3.000)$, a grey area (3.000-4.999) and a positive range ( $\geq 5.000)$ of hybridization signal intensities. Signal-to-background (S:B) values were calculated by dividing the signal intensity for each spot with the background signal intensities of the hybridized glass slide. Computer-assisted evaluation of the raw data provided the mean signal intensity and the signal:background ratio for each individual gene spot. For statistical evaluation, the mean signal intensity and standard deviation (SD) was calculated for each spot from the values obtained in the 10 individual experiments. Functional grouping of genes was performed on the basis of the database supplied by the array manufacturer.

\section{Results}

Mesenchymal stem cells

Microarray gene expression analysis

Integrins. The expression level and the change in expression of 9832 individual human genes in bone marrow-derived MSC was estimated on the basis of the hybridization signal intensity of each gene-specific probe represented by the microarray system. In the first series of microarray investigation, the gene expression in undifferentiated MSC was compared to the gene expression after chondrogenic differentiation in three paired experiments.

The MSC in their undifferentiated state expressed the components of the fibronectin-receptor $(\beta 1 \alpha 5)$ and downregulated these genes during chondrogenic differentiation (ratio Undiff:Diff, 0.58 for $\beta 1$ and 0.93 for $\alpha 5$; Fig. 1, Table I). Fibronectin was constantly expressed by MSC and during chondrogenic differentiation (ratio Undiff:Diff, 0.99). The components of the GPIIb/IIIa-receptor were expressed by MSC, $\alpha 2 b$ was expressed constantly during chondrogenic differentiation, $\alpha 3$ was downregulated (ratio Undiff:Diff, 1.55 for $\alpha 2 b$ and 0.63 for $\alpha 3$ ). Integrin $\beta 3$ was not expressed by MSC and, during chondrogenic differentiation (ratio Undiff:Diff, 0.49), Integrin $\alpha \mathrm{V}$ was constantly expressed (ratio Undiff:Diff, 1.49). Integrin 35 was downregulated during chondrogenic differentiation (ratio Undiff:Diff, 0.68) and Integrin $\alpha 10$ was not expressed.

Integrin-associated proteins. ILK was inactivated during chondrogenic differentiation (ratio Undiff:Diff, 0.63), CD47 was constantly expressed (ratio Undiff:Diff, 1.02) and ICAP-1a was not expressed.

\section{Chondrocytes}

Microarray gene expression analysis. The expression level and the change in expression of 9832 individual human genes in chondrocytes isolated from the septal cartilage was estimated on the basis of the hybridization signal intensity of each genespecific probe represented by the microarray system. In the first series of microarray investigation, the gene expression in primary chrondrocytes (day 1 ) was compared to the gene expression after 5 and 20 days of cell culture (days 6 and 21, respectively) in five paired experiments.

Integrins. The genes for the components of the fibronectin receptor (integrin $\beta 1 \alpha 5$ ) were activated during dedifferentiation (Table II; $\beta 1$ day 6:day 1 ratio, 2.73; $\beta 1$ day 21:day 1 ratio, 3.47 ; $\alpha 5$ day 6 :day 1 ratio, $2.11 ; \alpha 5$ day 21 :day 1 ratio, 0.74$)$. Fibronectin was constantly expressed during dedifferentiation 
A

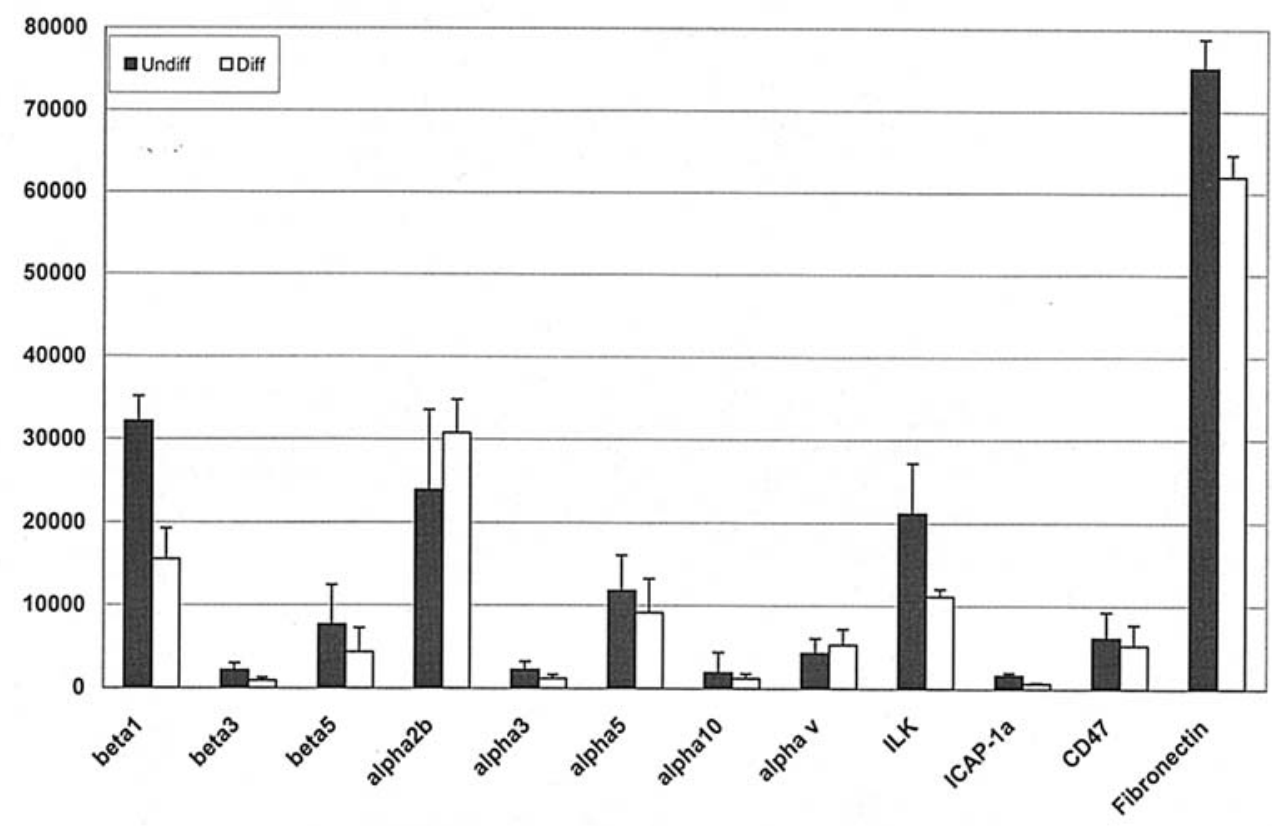

B

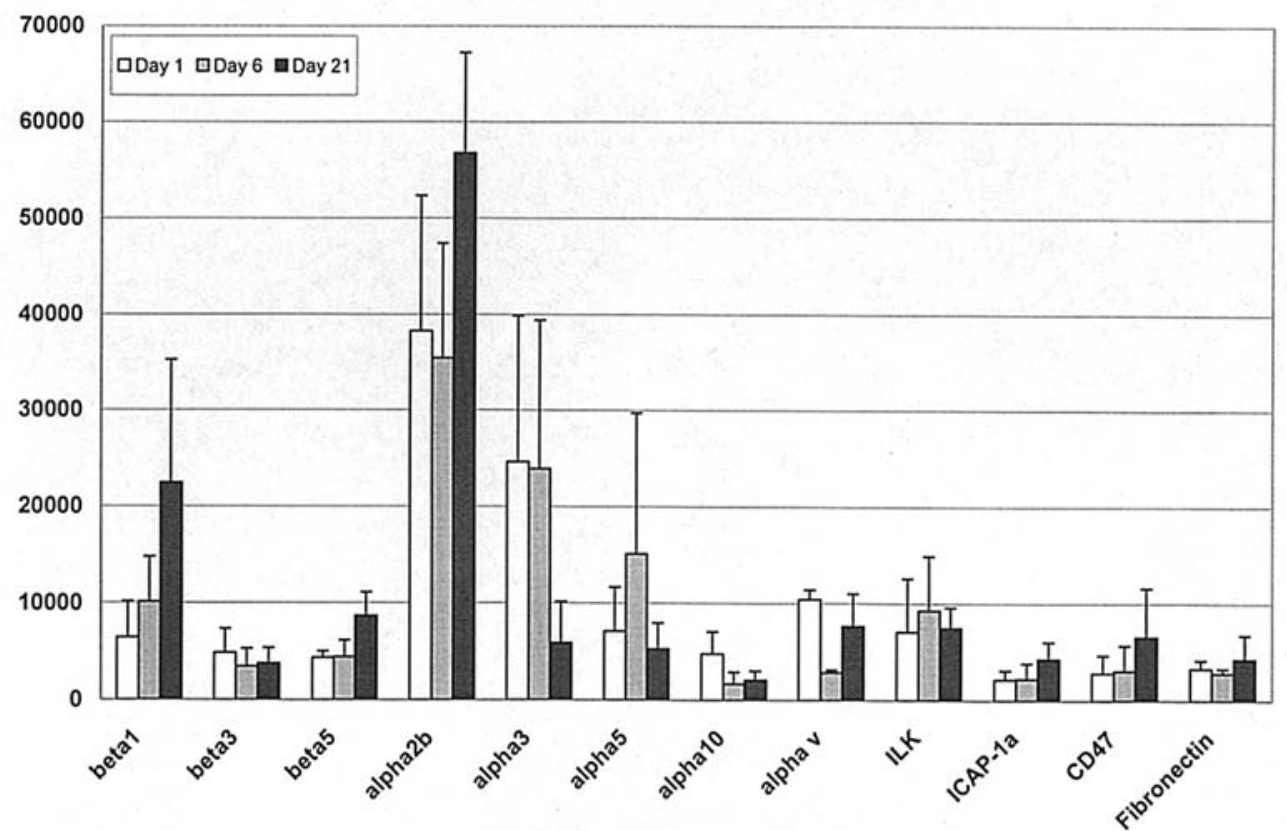

Figure 1. Expression levels of genes for different integrins and ECM-proteins in chondrocytes measured by microarray hybridization analysis. (A) Results from primary chondrocytes in vitro during dedifferentiation. Results are shown as mean signal intensity and standard deviation for the given genes in differentiated chondrocytes (white bars) on day 1, short-term cultured cells (grey bars) on day 6 and long-term cultured cells (black bars) on day 21. (B) Results from mesenchymal stem cells (MSC) during chondrogenic differentiation for the given genes in undifferentiated MSC (black bars) and chondrogenic differentiated MSC (white bars).

(day 6:day 1 ratio, 0.87; day 21:day 1 ratio, 1.3). The gene for integrin $\alpha 2 b$ was expressed on days 1, 6 and 21 (day 6:day 1 ratio, 0.92 ; day 21:day 1 ratio, 1.49$)$. The gene for integrin $\alpha \mathrm{v}$ was activated on days 1 and 21 but inactivated on day 6 (day 6:day 1 ratio, 0.28 ; day 21:day 1 ratio, 0.74 ). The integrin $\alpha 3$-gene was activated on days 1 and 6 and then downregulated on day 21 (day 6:day 1 ratio, 1.04; day 21:day 1 ratio, 0.24). The gene for integrin 33 was expressed in the grey area with a day 6:day 1 ratio of 0.95 and a day 21:day 1 ratio of 0.78 . The gene of integrin 85 was expressed on days 1 and 6 (day 6:day 1 ratio, 1.07) and was then activated on day 21 (day 21:day 1 ratio, 1.84 ). The genes for integrins $\beta 2$, $\beta 4, \beta 6, \beta 7$ and $\beta 8$ were never expressed. Integrin $\alpha 10$ was expressed on day 1 but then inactivated on days 6 and 21 (day 6:day 1 ratio, 0.35 ; day 21:day 1 ratio, 0.44 ) The genes for vitronectin and osteopontin were never expressed. 
Table II. Signal intensities of hybridization signals as measured by the microarray laser scanner and calculated by ArrayVision software during dedifferentiation of HC.

\begin{tabular}{|c|c|c|c|c|c|c|}
\hline & \multicolumn{3}{|c|}{ Signal intensity (mean \pm SD) } & \multicolumn{3}{|c|}{ Factor of regulation } \\
\hline & Day 1 & Day 6 & Day 21 & Day 1 vs. day 6 & Day 1 vs. day 21 & Day 6 vs. day 21 \\
\hline$\beta 1$ & $6465 \pm 3794$ & $10128 \pm 4640$ & $22418 \pm 12814$ & 2.73 & 3.47 & 0.45 \\
\hline$\beta 3$ & $4868 \pm 2506$ & $3481 \pm 1849$ & $3800 \pm 1600$ & 0.95 & 0.78 & 0.92 \\
\hline$\beta 5$ & $4378 \pm 675$ & $4463 \pm 1738$ & $8714 \pm 2401$ & 1.07 & 1.84 & 0.51 \\
\hline$\alpha 2 b$ & $38256 \pm 14115$ & $35463 \pm 11494$ & $56775 \pm 10340$ & 0.92 & 1.49 & 0.62 \\
\hline$\alpha 3$ & $24622 \pm 15239$ & $23926 \pm 15483$ & $5918 \pm 4294$ & 1.04 & 0.24 & 4.04 \\
\hline$\alpha 5$ & $7150 \pm 4522$ & $15126 \pm 14590$ & $5311 \pm 2697$ & 2.11 & 0.74 & 2.84 \\
\hline$\alpha 10$ & $4789 \pm 2302$ & $1717 \pm 1203$ & $2128 \pm 912$ & 0.35 & 0.44 & 0.81 \\
\hline$\alpha \mathrm{v}$ & $10476 \pm 961$ & $2903 \pm 965$ & $7706 \pm 3346$ & 0.28 & 0.74 & 0.38 \\
\hline ILK & $7081 \pm 5523$ & $9330 \pm 5609$ & $7508 \pm 2127$ & 1.32 & 1.06 & 1.24 \\
\hline ICAP-1a & $2192 \pm 910$ & $2245 \pm 1618$ & $4311 \pm 1742$ & 1.02 & 2.32 & 0.52 \\
\hline CD47 & $2835 \pm 1856$ & $3087 \pm 2641$ & $6582 \pm 5043$ & 1.09 & 1.97 & 0.47 \\
\hline Fibronectin & $3301 \pm 877$ & $2874 \pm 434$ & $4296 \pm 2488$ & 1.83 & 4.06 & 0.67 \\
\hline
\end{tabular}

Bold indicates significant up- $(\geq 2.00)$ or downregulation $(\leq 0.50)$.

Integrin-associated proteins. The fibronectin-gene was expressed on day 1 and then upregulated on days 6 and 21 (day 6:day 1 ratio, 1.83; day 21:day 1 ratio, 4.06). The gene for ILK was expressed on days 1, 6 and 21 with ratios of 1.32 and 1.06 respectively. Integrin ae, CD47 and integrin cytoplasmic domain-associated protein 1 (ICAP-1) were not expressed on day 1 but were activated on days 6 and 21 (day 6:day 1 ratios, 1.03, 1.09 and 1.02; day 21:day 1 ratios, 2.5, 2.32 and 1.97).

\section{Discussion}

Tissue engineering of cartilage comprises the creation of tissue grafts with autologous chondrocytes, an artificial extracellular matrix and biodegradable polymers. In past years, there have been several investigations with promising results which performed in vitro and in vivo maturation of tissue-engineering transplants for auricular (25) and nasal (26) cartilage. An important task of artificial breeding of tissue is to extract sufficient amounts of cells and to minimize donor site morbidity. So, one important task of tissue engineering is the efficient extraction of vital chondrocytes and adequate expansion (27). During monolayer culture, the chondrocytes dedifferentiate and lose their chondrocytic phenotype which is reflected on the molecular level by a repression of cartilagespecific collagen type 2 and an induction of type 1 collagen, typical for fibroblastoid cells (28). So far, no investigation of gene-expression patterns of chondrocytes has been performed. In the present study, characteristic changes of the expression of integrins and integrin-associated proteins were analyzed during dedifferentiation of primary chondrocytes in comparison with the expression during chondrogenic differentiation of mesenchymal stem cells.

The physiological response of chondrocytes to maintenance of the matrix and response to stimuli probably involves signaling from multiple sources including soluble cytokines, mechanical stimulation and signaling from the extracellular matrix. Cell-matrix interactions are important regulators of cellular functions, including matrix synthesis, proliferation and differentiation. This is well exemplified by the characteristically labile phenotype of chondrocytes that is lost in monolayer culture but is stabilized in suspension under appropriate conditions.

During dedifferentiation of the chondrocytes, the components of the fibronectin receptor, integrin $\$ 1 \alpha 5$, were activated. In contrast to these findings, the integrins $\beta 1 \alpha 5$ were downregulated during chondrogenic differentiation of MSC.

It has been previously described that, in primary chondrocytes, the most abundant integrin is the fibronectin-receptor, $\alpha 5 ß 1$ (29), which can also bind collagen 2, and that chondrocytes activate collagen type 2 during dedifferentiation. The upregulation of integrins $\beta 1 \alpha 5$ seems to correlate with these results, as upregulation would facilitate adhesion to collagen 2 . Integrins $\beta 1$ and $\alpha 5$ could be markers for synthetic activity as the chondrocytes try to generate an extracellular matrix suitable for their survival. Along with this activation of the fibronectin receptor, an upregulation of fibronectin in the course of dedifferentiation could be observed. Concordant with these results, the analysis of the gene expression of MSC during chondrogenic differentiation revealed a downregulation of integrins $\beta 1 \alpha 5$. So, on the one hand, the fibronectin receptor might be a marker for more undifferentiated, fibroblastoid cells (dedifferentiating chondrocytes, undifferentiated MSC) and, on the other hand, this receptor might assist the process of dedifferentiation.

Other receptors have been described for vitronectin and osteopontin $(\alpha \mathrm{V} \beta 3)$ or laminin $(\alpha 6 \beta 1)(30,31)$. We found expression of integrin $\alpha \mathrm{V}$ on day 1, downregulation on day 6 and upregulation on day 21. The gene for integrin 33 was 
expressed in the grey area and the gene for integrin 35 was upregulated on day 21. During chondrogenic differentiation of MSC, the behavior was vice versa: Integrins $B 3$ and $B 5$ were downregulated and integrin $\alpha \mathrm{V}$ was inactivated. Integrin 35 might play a role in vitronectin binding, as McLean and coworkers isolated cDNA clones coding for the B-5 subunit of the novel integrin receptor involved in cell adhesion to the matrix protein, vitronectin, which had been found in a human lung epithelial-derived cell line. This receptor had an $\alpha$ subunit that appeared to be identical to that in another vitronectin receptor, but the $\beta$ subunit was distinctive (32). In the present study, neither vitronectin nor osteopontin expression was observed, so these proteins do not seem to be involved in the dedifferentiation process.

In human chondrocytes, Camper and coworkers identified an additional type II collagen-binding integrin composed of $\beta-1$ and a $160-k D$ protein that they designated $\alpha-10$. We found downregulation of this integrin on days 6 and 21 and MSC downregulated both, integrins $\beta 1$ and $\alpha 10$, during chondrogenic differentiation. Therefore, the chondrocytes may switch mainly to integrin $\alpha 5 \beta 1$ with ongoing dedifferentiation (33).

The strong expression of Integrin $\alpha 2 b$ during dedifferentiation of $\mathrm{HC}$ was unexpected. Interestingly, there was upregulation of the $\alpha 2 \mathrm{~b}$-gene during chondrogenic differentiation of MSC and inactivation of the $\alpha 3$-gene. The Integrin $\alpha 2 b$ (ITGA2B) gene encodes platelet glycoprotein IIb, the $\alpha$ subunit of the platelet membrane adhesive protein receptor complex GPIIb/IIIa. The $\beta$ subunit, GPIIIa, is encoded by the Integrin $B 3$ gene. The GPIIb/IIIa complex belongs to the integrin class of cell adhesion molecule receptors that share a common heterodimeric structure with $\alpha$ and $\beta$ subunits. The GPIIb/GPIIIa complex mediates platelet aggregation by acting as a receptor for fibrinogen. The complex also acts as a receptor for fibronectin (19). Integrin $\alpha 2 b$ expression seems to be more important in chondrocyte dedifferentiation as the $\beta 3$ subunit. Possibly there is involvement of another $\beta$ subunit in the assembly of the receptor.

Integrin $\alpha 3$ showed strong expression on days 1 and 6 , but downregulation on day 21 with ongoing dedifferentiation of the cells. It has been shown previously that neuronal migration and layer formation might be regulated through modulation of $\alpha-3-\beta-1$ integrin-mediated neuronal adhesion and migration (34). Our experiments suggest that integrin $\alpha 3-$ dependent signaling is abundant in differentiated chondrocytes but is unnecessary in dedifferentiated chondrocytes.

Certain candidate genes from the integrin family might be involved in transducing the signals mediating the dedifferentiation of the chondrocytes in monolayer cell-culture. We aimed to find candidate genes that might transduce the signal to an intracellular level.

One of these candidates was Integrin-linked kinase (ILK). Microarray analysis revealed that the gene for ILK was expressed on days 1, 6 and 21 during the dedifferentiation of $\mathrm{HC}$ and the gene was inactivated during the chondrogenic differentiation of MSC. Hannigan et al isolated a gene that interacts with the cytoplasmic domain of $\beta-1$ integrin. They found that ILK coimmunoprecipitated with B-1 integrin from cell lysates and that overexpression of ILK disrupted the cell architecture and inhibited adhesion to integrin substrates while inducing anchorage-independent growth in epithelial cells, suggesting that ILK regulates integrin-mediated signal transduction (20). The expression of this gene suggests that integrin-mediated signalling is involved in the process of dedifferentiation.

In concordance with ILK, ICAP1 and CD47 were upregulated $(21,22)$ during the dedifferentiation of $\mathrm{HC}$ and downregulated during the chondrogenic differentiation of MSC. These three proteins involved in integrin-mediated processes during cell adhesion, differentiation and migration were upregulated during ongoing dedifferentiation in concordance with upregulation of the key members of the integrin familiy, e.g. integrin $\beta 1$. This substantiates the thesis of integrin-mediated signalling being involved in the process of dedifferentiation.

In conclusion, in the present study, we analysed expression patterns of integrins and related ECM-proteins. One of the candidates for signal-transmission is the fibronectin-receptor (integrin $\alpha 5 \beta 1$ ) in conjunction with its ligand fibronectin. Other receptors, e.g. for vitronectin and osteopontin $(\alpha \mathrm{VB3})$ or laminin $(\alpha 6 \beta 1)$ or their ligands do not seem to be involved in signal transmission for dedifferentiation. The strong expression of the components of the GPIIb/IIIa-receptor was unexpected, the latter seeming to be involved in the process of dedifferentiation. Intracellularly, ILK, ICAP1 and CD47 might be involved in the transduction of integrin-dependent signals.

\section{References}

1. Younger EM and Chapman MW: Morbidity at bone graft donor sites. J Orthop Trauma 3: 192-195, 1989.

2. Czyz J, Wiese C, Rolletschek A, Blyszczuk P, Cross M and Wobus AM: Potential of embryonic and adult stem cells in vitro. Biol Chem 384: 1391-1409, 2003.

3. Goessler UR, Hormann $\mathrm{K}$ and Riedel F: Tissue engineering with adult stem cells in reconstructive surgery (Review). Int $\mathbf{J}$ Mol Med 15: 899-905, 2005.

4. Weissman IL: Translating stem and progenitor cell biology to the clinic: barriers and opportunities. Science 287: 1442-1446, 2000.

5. Heng BC, Cao T, Haider HK, Wang DZ, Sim EK and Ng SC: An overview and synopsis of techniques for directing stem cell differentiation in vitro. Cell Tissue Res 315: 291-303, 2004.

6. Heng BC, Cao T and Lee EH: Directing stem cell differentiation into the chondrogenic lineage in vitro. Stem Cells 22: 1152-1167, 2004.

7. Stock UA and Vacanti JP: Tissue engineering: current state and prospects. Annu Rev Med 52: 443-451, 2001.

8. Goessler UR, Hormann K and Riedel F: Tissue engineering with chondrocytes and function of the extracellular matrix (Review). Int J Mol Med 13: 505-513, 2004.

9. Oakes BW: Orthopaedic tissue engineering: from laboratory to the clinic. Med J Aust 180: S35-S38, 2004.

10. Goessler UR, Bugert P, Bieback K, Baisch A, Sadick H, Verse T, Kluter H, Hormann $\mathrm{K}$ and Riedel F: Expression of collagen and fiber-associated proteins in human septal cartilage during in vitro dedifferentiation. Int J Mol Med 14: 1015-1022, 2004.

11. Goessler UR, Bugert P, Bieback K, Huber K, Fleischer I, Hörmann $\mathrm{K}$ and Riedel F: Differential modulation of integrin expression in chondrocytes during expansion for tissue engineering. In vivo 19: 501-507, 2005.

12. Loeser RF, Carlson CS and McGee MP: Expression of beta 1 integrins by cultured articular chondrocytes and in osteoarthritic cartilage. Exp Cell Res 217: 248-257, 1995.

13. Hering TM: Regulation of chondrocyte gene expression. Front Biosci 4: D743-D761, 1999.

14. Hynes RO: Integrins: versatility, modulation, and signaling in cell adhesion. Cell 69: 11-25, 1992.

15. Giancotti FG and Ruoslahti E: Integrin signaling. Science 285: 1028-1032, 1999.

16. Albelda SM and Buck CA: Integrins and other cell adhesion molecules. FASEB J 4: 2868-2880, 1990. 
17. Salter DM, Hughes DE, Simpson R and Gardner DL: Integrin expression by human articular chondrocytes. Br J Rheumatol 31: 231-234, 1992

18. Lee JW, Qi WN and Scully SP: The involvement of beta1 integrin in the modulation by collagen of chondrocyte-response to transforming growth factor-beta1. J Orthop Res 20: 66-75, 2002.

19. Prandini MH, Denarier E, Frachet P, Uzan G and Marguerie G: Isolation of the human platelet glycoprotein IIb gene and characterization of the 5 ' flanking region. Biochem Biophys Res Commun 156: 595-601, 1988.

20. Hannigan GE, Leung-Hagesteijn C, Fitz-Gibbon L, Coppolino MG, Radeva G, Filmus J, Bell JC and Dedhar S: Regulation of cell adhesion and anchorage-dependent growth by a new beta 1-integrin-linked protein kinase. Nature 379: 91-96, 1996.

21. Bouvard D and Block MR: Calcium/calmodulin-dependent protein kinase II controls integrin alpha5beta1-mediated cell adhesion through the integrin cytoplasmic domain associated protein-1alpha. Biochem Biophys Res Commun 252: 46-50, 1998.

22. Mawby WJ, Holmes CH, Anstee DJ, Spring FA and Tanner MJ: Isolation and characterization of CD47 glycoprotein: a multispanning membrane protein which is the same as integrinassociated protein (IAP) and the ovarian tumour marker OA3. Biochem J 304: 525-530, 1994.

23. Mackay AM, Beck SC, Murphy JM, Barry FP, Chichester CO and Pittenger MF: Chondrogenic differentiation of cultured human mesenchymal stem cells from marrow. Tissue Eng 4: 415-428, 1998.

24. Bugert P, Dugrillon A, Gunaydin A, Eichler H and Kluter H: Messenger RNA profiling of human platelets by microarray hybridization. Thromb Haemos 90: 738-748, 2003.

25. Haisch A, Klaring S, Groger A, Gebert C and Sittinger M: A tissue-engineering model for the manufacture of auricular-shaped cartilage implants. Eur Arch Otorhinolaryngol 259: 316-321, 2002.
26. Rodriguez A, Cao YL, Ibarra C, Pap S, Vacanti M, Eavey MD and Vacanti CA: Characteristics of cartilage engineered from human pediatric auricular cartilage. Plast Reconstr Surg 103: 1111-1119, 1999.

27. Gruber R, Sittinger M and Bujia J: In vitro cultivation of human chondrocytes using autologous human serum supplemented culture medium: minimizing possible risk of infection with pathogens of prion diseases. Laryngorhinootologie 75: 105-108, 1996.

28. Benya PD and Shaffer JD: Dedifferentiated chondrocytes reexpress the differentiated collagen phenotype when cultured in agarose gels. Cell 30: 215-224, 1982.

29. Shakibaei M and Merker HJ: Beta1-integrins in the cartilage matrix. Cell Tissue Res 296: 565-573, 1999

30. Enomoto M, Leboy PS, Menko AS and Boettinger D: Beta 1 integrins mediate chondrocyte interaction with type I collagen, type II collagen, and fibronectin. Exp Cell Res 205: 276-285, 1993.

31. Durr J, Goodman S, Potocnik A, von der Mark H and von der Mark K: Localization of beta 1-integrins in human cartilage and their role in chondrocyte adhesion to collagen and fibronectin. Exp Cell Res 207: 235-244, 1993.

32. McLean JW, Vestal DJ, Cheresh DA and Bodary SC: cDNA sequence of the human integrin beta 5 subunit. J Biol Chem 265: 17126-17131, 1990.

33. Camper L, Hellman U and Lundgren-Akerlund E: Isolation, cloning, and sequence analysis of the integrin subunit alpha10, a beta1-associated collagen binding integrin expressed on chondrocytes. J Biol Chem 273: 20383-20389, 1998.

34. Dulabon L, Olson EC, Taglienti MG, Eisenhuth S, McGrath B, Walsh CA, Kreidberg JA and Anton ES: Reelin binds alpha3beta1 integrin and inhibits neuronal migration. Neuron 27: 33-44, 2000 